\title{
The electron diffractometer: A dedicated user-friendly device for electron crystallography experiments \\ Gustavo Santiso-Quinones' ${ }^{1}$, Arianna E. Lanza², Luca Piazza ${ }^{3}$, Eric Hovestreydt ${ }^{4}$, Christoph Hoermann $^{5}$, Clemens Schulze-Briese ${ }^{6}$ \\ ${ }^{1}$ Eldico Scientific AG ${ }^{2}$ ELDICO Scientific AG, ${ }^{3}$ DECTRIS Ltd. ${ }^{4}$ ELDICO Scientific AG, ${ }^{5}$ DECTRIS AG, ${ }^{6}$ DECTRIS Ltd \\ santiso@eldico.ch
}

Keywords: electron diffractometer; electron diffraction; electron crystallography; nanocrystallography, hybrid-pixel electron detector

3D electron diffraction experiments for structural chemistry and structural biology are undergoing a major leap in the context of the nanocrystallography revolution [1]. This is mainly due to the availability of highly sensitive, very fast and low-noise detectors.

Moreover, the continuous rotation method (known also as microED) for the collection of electron diffraction data is surpassing all previous data collection methods available in the past decade as it makes electron crystallography experiments and data analysis conceptually as straightforward as standard X-ray crystallography.

Unfortunately, the lack of dedicated instrumentation for performing electron diffraction experiments is hindering the accessibility of this powerful technique for the crystallographic community. All experiments found in the literature were in fact performed in (modified)-Transmission Electron Microscopes (TEMs), thus required specialized expertise, and methodological workarounds. TEM devices are not optimal for performing such kind of experiments and there is no standardized protocol for data collection, data analysis and/or validation yet.

As the interest in this technique is growing, scientists have developed their own protocols, methods and guidelines for using TEMs as an electron diffractometer [2-4]. In response to the high demand for a dedicated instrument, here we present the electron diffractometer, a novel device optimized for electron diffraction experiments using the continuous rotation method. Its ease of use makes it attractive for crystallographers and scientists.

We will showcase this device and its ease of use. Furthermore, we will highlight one of its core elements, a hybrid-pixel electron detector, DECTRIS QUADRO. Its unique characteristics such as zero readout noise, small point-spread function, high frame rate, and high dynamic range allow for better and faster data collection. Experimental results obtained using this device will be presented too.

[1] Gemmi, M. Mugnaioli, E. Gorelik, T. E. Kolb, U. Palatinus, L. Boullay, P. Hövmoller, S. and Abrahams, J. P. (2019). ACS Cent Sci. 5, 1315-1329.

[2] Heidler, J. Pantelic, R. Wennmacher, J. T. C. Zaubitzer, C. Fecteau-Lefebvre, A. Goldie, K. N. Müller, E. Holstein, J. J. van Genderen, E. De Carlo, S. \& Gruene, T. (2019). Acta Cryst. D75, 458-466.

[3] Nannenga, B.L. Gonen, T. (2019). Nat Methods, 16, 369-379.

[4] Roslova, M. Smeets, S. Wang, B. Thersleff, T. Xu, H. \& Zou, X. (2020). J. Appl. Cryst. 53, 1217-1224. 